\title{
Web-based Information System (WBIS) Framework: Facilitating Interoperability within Business Ventures
}

\author{
Adetunji Oluwatofunmi O. \\ Computer Science Department \\ Babcock University
}

\author{
Idowu S. A. \\ Computer Science Department \\ Babcock University
}

\author{
Izang A. A. \\ Computer Science \\ Department \\ Babcock University
}

\begin{abstract}
Business organizations need to keep abreast of relevant information so as to be updated within the economy as globalization of the world made it possible to get a great deal of information in less time. Having several business units belonging to the same business venture comes along with some disadvantages such as duplication of processes that cuts across all units, duplication of records stored within the database, increase in the cost of maintaining and securing the database as well as increase in the cost of maintaining and managing each unit. This therefore shows the need for having an interoperable system that can enable the management of all units from a single point. The main objective of this work is to develop a web-based Business Information System Framework which can be adopted by business owners to perform specific operations within their respective organizations. A framework was proposed using a business logic which ensured that business rule in the real world were adhered to. The proposed framework specifies two levels of managements - unit administrator which cater for the individual business unit and the central or super admin who manages all available units from a single point. The research work presents a framework that provide central administrators the ability to get relevant information that cut across all functional business units created by the Business Venture, this interoperable framework in turn helps in reducing complexities within Business Ventures.
\end{abstract}

\section{Keywords}

Business Information System, Business Venture, Business Unit, Business Process, Interoperable Framework.

\section{INTRODUCTION}

A system is defined as various collection of components that work together to achieve a common goal. According to [1] a system is a set of elements or components that interact with each other to achieve a particular goal. In reality, most system do not have a single goal - they constitute of sub systems with sub goals which individually contributes to the overall system goal [2]. The objective of a system is to accept input, manipulate or transform such inputs into output. Hence, good system is expected to have inputs, processing mechanism, outputs as well as feedback.

A Business Venture is an enterprise in which the expectation of gain is accompanied by the risk of loss or failure. Business ventures are being developed with the intent of making financial profit. Individuals or group invest in business ventures with the expectation of the business bringing financial gain. Business ventures is one which have several business units which often engage in different business ideas which might be stationed in different geographical locations it can be regarded to as a multi divisional organization whichcan be referred to as a firm with several semiautonomous units [3]. Business units of a business venture most time have unique computer software that manages each unit due to the peculiar nature of each unit, this means there are different software used within a business venture, each software having its' own database. This is more likely to happen when each units specializes in providing peculiar products and services to the community at large. Some Business Venture might use the same software across all units but lack central management as well as a centralized database due to the fact that the units are semi-autonomous.

It has been observed that the management of Business Ventures with several business units which specializes in the sales of different products have been very difficult due to the peculiar nature of each unit especially when these units are stationed at different locations. Many Business Venture operates as a non-centralized decision making organization which in turn increases the autonomy of its sub-units and abates the effectiveness of their systems' integration and interoperability. It is essential for systems within an enterprise or business organization to be interoperable in a business environment so as to enable seamless sharing of business information and knowledge.

Interoperability between individual business units and the central management has been discovered to be a major issue which prevents the Business Venture at large from making significant progress. Interoperability which is defined as the ability of two or more system components to communicate and exchange information and also make use of the information that has been exchanged has become a major challenge between autonomous business units that are connected to a mother enterprise. The keeping of records in a decentralized manner among business units has been seen as another challenge in Business Ventures due to the fact that the cost of maintaining and securing the database increases with respect to the number of functional units, data integrity is minimized while data redundancy is maximized.

The objective of the study is to propose an interoperable Web Based Information System (WBIS) framework for Business Ventures which can be adopted by business owners to perform specific operations within their respective organizations. This research work covered the design of an interoperable Web Based Information System (WBIS) framework for Business Ventures. The framework will enable 
the interoperability between the super administrator and the unit administrator and will accommodate both online customers who will make selection and payments of items without going to the store. This research work enables the interoperability of business units within the same Business Venture, it also allows central management of all units by a super administrator who from a single point can monitor the lower level administrators these units.

\section{LITERATURE REVIEW}

\subsection{Overview of Information System}

Systems can be simply be defined as a collection of several components that work together to achieve a common goal. A system can also be defined as a set of elements or components that interact to accomplish a particular goal [1]. Ralph stated that the elements of a system and the relationship that exist among them determines how the system works.

It was noted by [2] that not every system has a single goal or objective thereby making some system have several sub systems alongside with sub goals of which they all contribute to meeting the overall system goal. Every system is expected to have a form of input, processing mechanism, outputs, feedback and control mechanisms. Simply put, a system processes input (data) to create output (information), feedback mechanism are required in every system to maintain and monitor the performance, there must also be a control mechanism to correct problems and ensure that a system is fulfilling its purpose.

The effective and efficient management of information can in this present generation be considered as an integral part of an organization which make people and organization make use of information on a daily basis. Business Organizations in today's world make use of Information Systems to increase revenue and reduce cost [1], Information System involve the use of computers and are therefore changing the way in which organizations carry out their businesses, it provides tangible information to management in order to enable them make decisions which will help in keeping the organization in check thereby supporting the operational management and decision making process within the organization [4].

Information System is one the discipline in the academia that lacks a unified definition which is serving as one of the major obstacles troubling the field of Information System [5], this challenge was also identified by [6] .

\subsection{Overview of Business Information}

\section{System}

Information has become so vital in today's world such that it is needed for varied number of reasons. Information are gotten from diverse places such as watching television, reading newspapers, books, magazines, surfing the internet as well as listening to the radio. All these activities are being carried out by humans so as to remain enlightened and aware of the environment.

In the business world, various individuals and organizations require lots of information in order to make sound and effective decisions so as to solve lots of problems [7]. Sousa and $\mathrm{Oz}$ identified making sound decisions and solving problems as the two major closely related practices that form the foundation of any successful company. Most organizations and businesses aim at being the market leaders in their various industries amidst several challenging factors such as recession, inflation and so on. [8] in an online article stated that businesses must adopt the information systems so as to stay ahead of others in the industry, as information systems help companies to make maximum and adequate use of its data, reduces work load and also assist companies in complying to various mandatory rules and regulations.

One of the impact of globalization in the business world is the possibility of getting greater amount of information in much less time which means that companies are thereby required to spend much of their time and energy in handling the increase information.

Business Information System according to [9]is a group of interrelated components that work collectively to carry out input, processing, output, storage and control actions in order to transform data into tangible information that can be used in forecasting, coordinating decision making and operational activities within an organization.

Business Information System provides suitable IT solutions thereby supporting people and their business processes [10], it help to foster companies competitive advantage by providing support and for making timely decisions because they are mainly concerned with information and decision inputs.

\subsection{Overview of Business Venture}

It is obvious that humans have needs that ought to be satisfied. In a bid to meet this needs, some members of the society need to engage in continuous activities so as to guarantee the satisfaction of others - this can be termed 'business'. Business can therefore be defined as an economic activity which involve continuous and regular production and distribution of goods and services to satisfy human wants [11].

[12] defined the term business means as a persistent distribution and production of services and products to consumer under uncertain market conditions. Businesses are predominant in capitalist economies where most of the businesses are privately owned and administered to earn profit and also to increase the wealth of the business owners. A business venture is a start-up enterprise that is formed with the expectation and plan that financial gain will be realized [13]. Generally speaking, an attempt to meet the needs of the people in an economy give rise to Business Ventures which provide people with products or services.

A Business Venture can also be referred to as a business enterprise or a Business Organization with one or more business units attached to it. The whole Business Organizations is controlled by a central management but most decisions are taken by each autonomous division.

\subsection{Review of Related Works}

[14] designed and implemented a Management Information System Based on VC++ in which Microsoft SQL Server was the database which was used while the Visual $\mathrm{C}++$ was the programming language of the designed system. The weakness of this research was found in the methodology used. Microsoft SQL Server is not a good database for Information Systems due to the large number of Information which is always stored and processed by most Information Systems. The programing language used (Visual $\mathrm{C}++$ ) has lots of disadvantages which made the system inefficient in today's world. Some challenges found with the choice of programing language are that it possess a non-standard implementation and is not 
suitable for cross platform coding - it is not standard compliant thus the source code may not be portable unless a conscious effort is made to maintain portability by sticking to standards that are not supported.

[15] came up with a Management information System for Densu Technology Company. The designed system was based on ASP.NET, Business Information System Development Model and a database technology. The system was divided into two modules which are: Customer Module and the Management Module. The aim of the research work was to develop an efficient way in which information could be delivered to the customer. On the other hand, it provided an online multimedia editing platform for the administrators to enter text, insert images, tables and so on which will automatically be converted and saved as an HTML file. A major pitfall of this research is that only a single Business Organizations was considered and used in the implementation. The designed system is not flexible such that it can be used and customized by other Business Organizations. [16] implemented a Business Information System Framework for Restaurants. The iterative software methodology was used, the programing Language used was PHP and the Database used was MySQL. The pitfall identified in this research work was that it considered only a portion of a Business Venture (Restaurant) in a single location without putting into consideration the probability of the business organisation expanding to multiple locations and also the probability of the business organization creating other business sub units which might likely specialize in other products or service.

[17] based on web services designed and implemented a Management information System for product logistics. The research presented a software architecture design methods and implementation techniques for a web service logistic information system which was based on browser, web server, application server, and data structure. This system was divided into three layers which are the User Interface Layer, Business Logical Layer and the Data Access Layer. The design system included the following subsystems or modules: Business Management Warehouse Management, Distribution Management, Accounting Management and Customer Service.
The sole purpose of the system was to keep track of logistics information of products for a particular business type. However this system does not provide the management or decision makers with information needed to make tangible decisions. It was developed using the following technologies: SQL Server 2008 as the database, ASP.NET was the chosen programing language while Microsoft Information Services (IIS) was used as the web server. The choices of the technology adopted posed some pitfalls of the developed system such as the database used was proprietary which will not enable the development of an open source software, SQL Server 2008 does not go along well with other programing languages other than the '.NET Framework' programing languages. It was stated by [18] that SQL Server is not suitable for applications with large and scalable. The web server used (IIS) lacks flexibility - it does not provide a way to control how Uniform Resource Locators (URLs) are parsed of which it can only be used in the windows operating system environments [19]

Based on the review of the related works, this research work will therefore create a business information system framework which will enable interoperability between sub units and the central management of a Business Venture thus providing business owners or super administrators the ability to monitor the entire venture from a single point and also create online stores at will, feedback mechanism will also be implemented to enable customers send complaints to the management.

\section{METHODOLOGY}

The interoperable framework was presented with a business logic which ensured that the business rules in the real world were properly encoded. This was done after the review of several existing frameworks. The framework specifies two level managements. The first which is the administrator of individual business units who submits and is subjected to the super and central administrator who oversees all the activities of all business units. The business logic layer ensures that the business rules in the real world are being encoded, it therefore determines how data can be created, displayed, stored and changed.

\section{PROPOSED FRAMEWORK}

Figure 1 shows the proposed framework. 


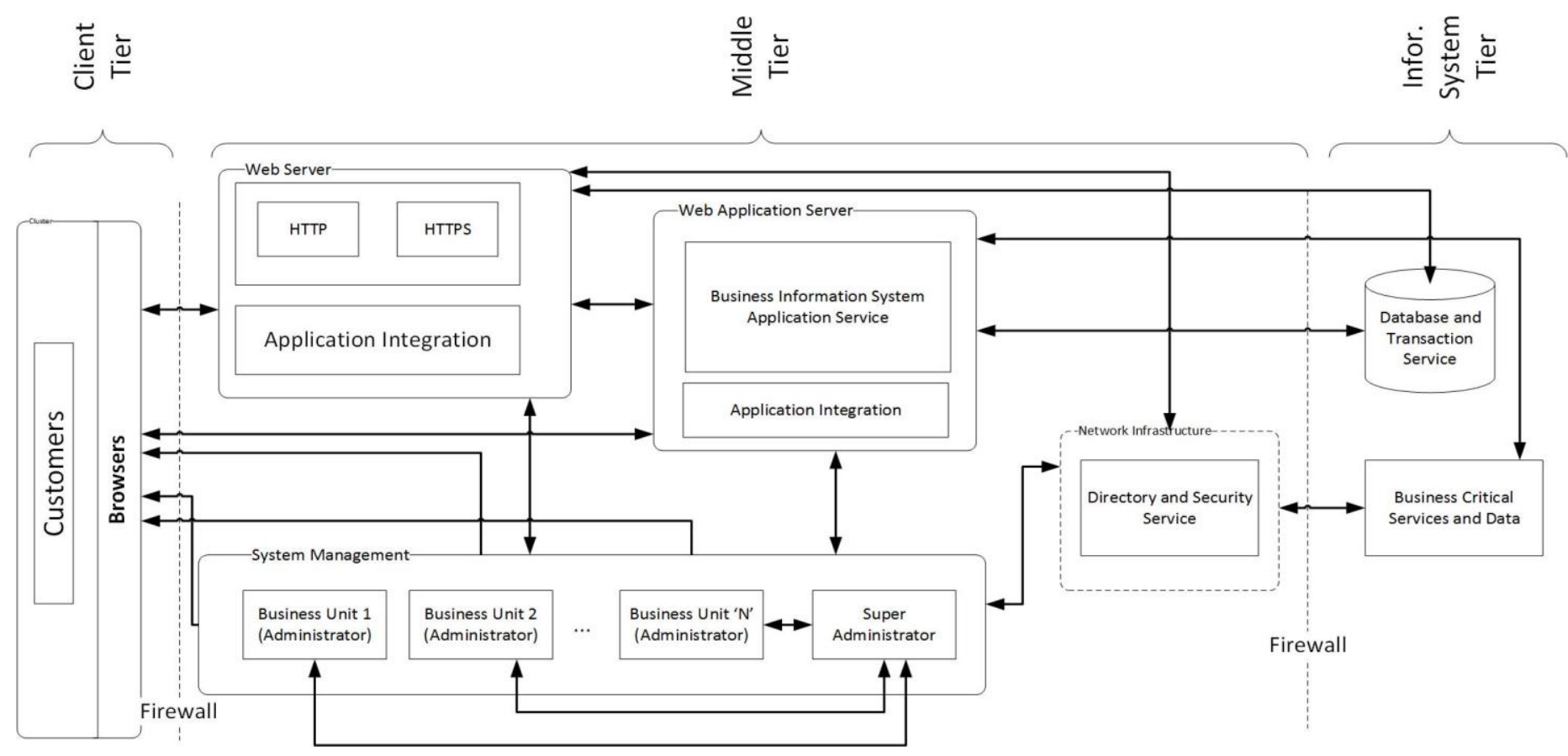

Fig 1: Architectural Diagram for the proposed WBIS Framework

\subsection{Information System Architecture}

The framework architecture structures the available services for a Business Venture application. It defines the relationship between various parts and services and constitutes the following key elements.

1. Servers (Web Server/Web Application): This servers together provide the main business logic and presentation services for the Business Venture application. The services being provided includes: Database and Transaction Services, Mailing Services, Collaboration Services (used for collaboration between the Super Administrator, Business Unit Administrator and the Customers) and other related services.

2. Clients: This includes customers using devices which ranges from standard web browsers to pervasive devices such as personal digital assistants, smart phones and so on.

3. Application Integration Software: Which enables access and integration to existing data.

4. Network Infrastructure: This provides network connectivity, directory and security services.

5. System Management: This help to accumulate the unique structure of management that will be adopted by the Business Venture application.

6. Business Information System Application Services: This provides prebuilt building blocks which will help to facilitate the creation of the Business Venture solution.

7. Tools: This help to build, run and manage the Business Venture solution.

Details of the element listed above are given below.

\subsection{Web Application Server}

The application server within the framework exposes the business logic to client application through various protocols, it provide the core services required to develop and also to support the presentation and business logic of the Business Venture application. The application server includes the Web Server, Database Services, Messaging Services, Mailing Services and Collaboration Services.

1. Web Server: This handles the HTTP protocol - it receives HTTP requests and responds to the request. It coordinates the collection and assembly of web pages ranging from static to dynamic contents and then delivers it to the clients. When a request comes into the webserver, the web server then passes the request to a program which can handle it. In simple terms, it provides an environment in which a serverside program can execute a request and then pass back the generated response.

2. Database Service: This provides the framework application with the features and functions of a relational database. This service provides query, update, delete and insert access to all users of the system (depending on the users' role) through the use of a Structured Query Language - MySQL.

3. Mailing Service: This service enables the Business application to have a closer relationship with its customers, partners and the in-house team members. The main services provided includes: Email Messaging, News Group Discussions.

This service is based on the protocols for internet technology such as the Simple Mail Transfer Protocol (SMTP) or the Internet Messaging Access 
Protocol (IMAP) for sending and receiving mails and the Network News Transfer Protocol (NNTP) for the news group discussions.

4. Collaborative Services: this help to provide what is known as a virtual work place to support the coordination and workflow of the business.

\subsection{Application Integration}

This is located in the middle tier of the framework. It provides the framework with the ability to access existing computing assets such as the existing database, it also provide a means in which the framework can intelligently integrate business process that are built on disparate computing architecture.

\subsection{Clients}

This includes a wide range of devices from personal computers to smart phones, personal digital assistants and other reliable information appliances. Due to the distinct capabilities of the above mentioned appliances which vary significantly, they have to rely on certain sets of web based technologies to unify the response gotten from both the webserver and the application server.

\subsection{Network Infrastructure}

This provides the framework with a secure, reliable networking platform. Based on the open standards, the infrastructure includes the network services which supports the Business Venture applications internet, intranet and extranet. It provides security services that stops unauthorized access across the network environment and also includes a directory service which is used to locate users, services as well as resources in the network.

\subsection{System Management}

A network centric systems management services and tool is included in the framework. The system management is used to manage the entire application and it is made up of Business Unit Administrators which are all connected to the super administrator in which they monitor and control the day to day activities in a business unit. The super administrator who oversees the activities of the entire Business Venture.

The system management services is connected to all other services in the framework and also supports the management of the complete life of the Business Venture application.

\subsection{WBIS Framework}

The WBIS framework specifies two level of managements. The first which is the administrator of individual business units who submits and is subjected to the super or central administrator who oversees all the activities of all business units. The business logic layer ensures that the business rules in the real world are being encoded, it therefore determines how data can be created, displayed, stored and changed. Figure 2 shows the WBIS interoperable framework.

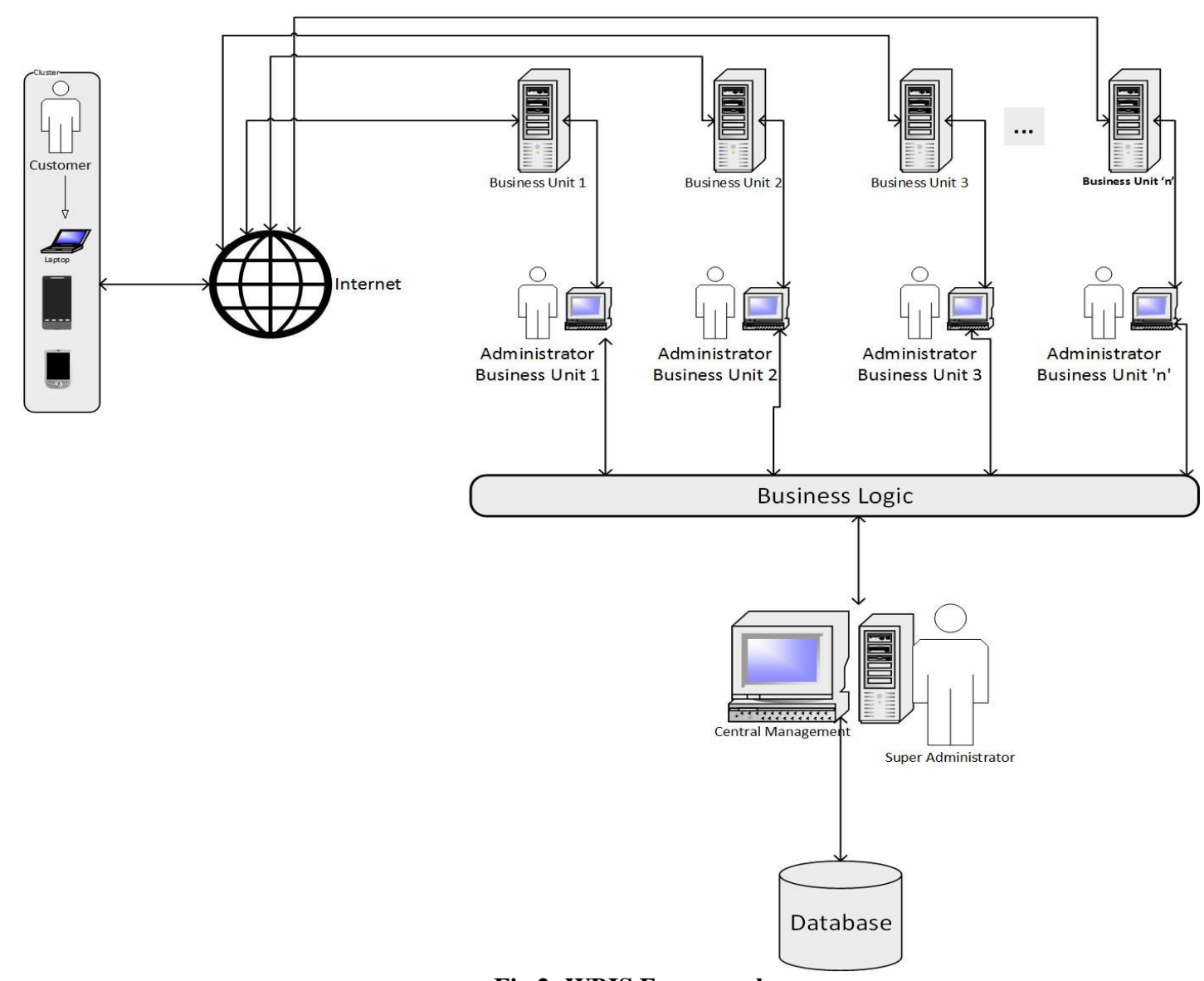

Fig 2: WBIS Framework 


\section{SUMMARY AND CONCLUSION}

The need for Information System in an organization was emphasized in which the globalization of the economy was pinpointed as the main reason why information grow at a rapid rate. Keeping track of Business Units which springs up from a mother Business Venture is seen as a difficult task especially when this sub units are sited in different geographical locations. However, there was the need to develop an interoperable framework that can be used to monitor and keep track of these units. This led to the design of a WBIS Framework for Business Ventures.

\section{REFERENCES}

[1] S. Ralph, R. George, and C. Thomas, Fundamentals of Business Information Systems, 2 ed.: Andrew Ashwin, 2012.

[2] H. Elizabeth, Business Information Systems, 2 ed.: Elizabeth Hardcastle \& Book Boon, 2011.

[3] O. Furrer, Corporate level strategy: Theory and applications: Routledge, 2016.

[4] S. Bulgacs, "The first phase of creating a standardised international innovative technological implementation framework/software application," International Journal of Business and Systems Research, vol. 7, pp. 250-265, 2013.

[5] S. Alter, "Defining Information Systems as Work Systems: Implications for the IS Field," European Journal of Information Systems, vol. 17, pp. 448469, October 2008.

[6] R. J. Paul, "Challenges to information systems: time to change," EJIS, vol. 16, pp. 193-195, 2007.

[7] K. J. Sousa and E. Oz, Management Information Systems: Cengage Learning, 2014.

[8] J. Davoren. (2016, 18/08). The Three Fundamental Roles of Information Systems in Business. Available: http://smallbusiness.chron.com/threefundamental-roles-information-systems-business23681.html

[9] K. C. Laudon and J. P. Laudon, Essentials of Business Information Systems, 7 ed. New Jersy: Pearson Education, 2007.

[10] J. Rubart, "Semantic Adaptation of Business Information Systems using Human-Centered
Business Rule Engines," in 2016 IEEE Tenth International Conference on Semantic Computing (ICSC), 2016, pp. 187-193.

[11] G. Akrani. (2011, 25/08). What is Business? Meaning Definitions Features of Business Available: http://kalyancity.blogspot.com.ng/2011/03/what-is-businessmeaning-definitions.html

[12] F. Masoumigoudarzi, "Business value modeling based on BPMN models," 2014

[13] WiseGeek. (2016, 25/08/2016). What is a Business Venture? Available: http://www.wisegeek.com/what-is-a-businessventure.htm

[14] S. Peimin, "Design and implementation of management information system based on $\mathrm{VC}++, "$ in E-Health Networking, Digital Ecosystems and Technologies (EDT), 2010 International Conference on, 2010, pp. 480-483.

[15] Z. Liping and D. Hualing, "The Design and Implementation of Management Information System for Dentsu Technology Company," in 2011 International Conference on Information Management, Innovation Management and Industrial Engineering, 2011, pp. 239-242.

[16] T. Slinger, "A Business Information System Framework for Restaurants," University of Leeds, School of Computing Studies, 2011.

[17] H. Jiang, Y. Li, and H. Fang, "Design and Implementation of Logistics Information Management System Based on Web Service," in 2015 14th International Symposium on Distributed Computing and Applications for Business Engineering and Science (DCABES), 2015, pp. 130133.

[18] Dickyibrohim. (2016, 05/09/2016). Advantages and Disadvantages of Microsoft SQL Server. Available: $\mathrm{http} / / / \mathrm{blog}$.cleoraa.com/advantages-anddisadvantages-of-microsoft-sql-server/

[19] P. Hilton. (2014, 05/09). Pros and cons of using IIS, ASP, PerlScript and ADO on Windows NT Available: http://hilton.org.uk/iis-asp-perlscript-ado 